\title{
Efficient CNF Simplification based on Binary Implication Graphs ${ }^{\star}$
}

\author{
Marijn Heule $^{1}$, Matti Järvisalo ${ }^{2}$, and Armin Biere ${ }^{3}$ \\ 1 Department of Software Technology, Delft University of Technology, The Netherlands \\ 2 Department of Computer Science, University of Helsinki, Finland \\ ${ }^{3}$ Institute for Formal Models and Verification, Johannes Kepler University Linz, Austria
}

\begin{abstract}
This paper develops techniques for efficiently detecting redundancies in $\mathrm{CNF}$ formulas. We introduce the concept of hidden literals, resulting in the novel technique of hidden literal elimination. We develop a practical simplification algorithm that enables "Unhiding" various redundancies in a unified framework. Based on time stamping literals in the binary implication graph, the algorithm applies various binary clause based simplifications, including techniques that, when run repeatedly until fixpoint, can be too costly. Unhiding can also be applied during search, taking learnt clauses into account. We show that Unhiding gives performance improvements on real-world SAT competition benchmarks.
\end{abstract}

\section{Introduction}

Applying reasoning techniques (see e.g. [1234567]) to simplify Boolean satisfiability (SAT) instances both before and during search is important for improving stateof-the-art SAT solvers. This paper develops techniques for efficiently detecting and removing redundancies from CNF (conjunctive normal form) formulas based on the underlying binary clause structure (i.e., the binary implication graph) of the formulas.

In addition to considering known simplification techniques (hidden tautology elimination (HTE) [6], hyper binary resolution (HBR) [17], failed literal elimination over binary clauses [8], equivalent literal substitution [89]10], and transitive reduction [11] of the binary implication graph [10]), we introduce the novel technique of hidden literal elimination (HLE) that removes so-called hidden literals from clauses without affecting the set of satisfying assignments. We establish basic properties of HLE, including conditions for achieving confluence when combined with equivalent literal substitution.

As the second main contribution, we develop an efficient and practical simplification algorithm that enables "Unhiding" various redundancies in a unified framework. Based on time stamping literals via randomized depth-first search (DFS) over the binary implication graph, the algorithm provides efficient approximations of various binary clause based simplifications which, when run repeatedly until fixpoint, can be too costly. In particular, while our Unhiding algorithm is linear time in the total number of literals (with an at most logarithmic factor in the length of the longest clause), notice as an example that fixpoint computation of failed literals, even just on the binary implication

\footnotetext{
* The $1^{\text {st }}$ author is financially supported by Dutch Organization for Scientific Research (grant 617.023.611), the $2^{\text {nd }}$ author by Academy of Finland (grant 132812) and the $1^{\text {st }}$ and $3^{\text {rd }}$ author are supported by the Austrian Science Foundation (FWF) NFN Grant S11408-N23 (RiSE).
} 
graph, is conjectured to be at least quadratic in the worst case [8]. Unhiding can be implemented without occurrence lists, and can hence be applied not only as a preprocessor but also during search, which allows to take learnt clauses into account. Indeed, we show that, when integrated into the state-of-the-art SAT solver Lingeling [12], $U n$ hiding gives performance improvements on real-world SAT competition benchmarks.

On related work, Van Gelder [8] studied exact and approximate DFS-based algorithms for computing equivalent literals, failed literals over binary clauses, and implied (transitive) binary clauses. The main differences to this work are: (i) Unhiding approximates the additional techniques of HTE, HLE, and HBR; (ii) the advanced DFS-based time stamping scheme of Unhiding detects failed and equivalent literals on-the-fly, in addition to removing (instead of adding as in [8]) transitive edges in the binary implication graph; and (iii) Unhiding is integrated into a clause learning (CDCL) solver, improving its performance on real application instances (in [8] only random 2-SAT instances were considered). Our advanced stamping scheme can be seen as an extension of the BinSATSCC-1 algorithm in [13] which excludes (in addition to cases (i) and (iii)) transitive reduction. Furthermore, while [13] focuses on simplifing the binary implication graph, we use reachability information obtained from traversing it to simplify larger clauses, including learnt clauses, in addition to extracting failed literals.

As for more recent developments, CryptoMiniSAT v2.9.0 [14] caches implied literals, and updates the cache after top-level decisions. The cache can serve a similar purpose as our algorithms, removing literals and clauses. Yet, the cache size is quadratic in the number of literals, which is also the case for using the cache for redundancy removal for the whole CNF. Thus, at least from a complexity point of view, the cache of CryptoMiniSAT does not improve on the quadratic algorithm [8]. In contrast, Unhiding requires only a single sweep over the binary implication graph and the other clauses.

After preliminaries (CNF satisfiability and known CNF simplification techniques, Sect.2), we introduce hidden literal elimination and establish its basic properties (Sect. 3). We then explain the Unhiding algorithm: basic idea (Sect. 4) and integration of simplification techniques (Sect. 5). Then we develop an advanced version of Unhiding that can detect further redundancies (Sect.6), and present experimental results (Sect.77).

\section{Preliminaries}

For a Boolean variable $x$, there are two literals, the positive literal $x$ and the negative literal $\bar{x}$. A clause is a disjunction of literals and a CNF formula a conjunction of clauses. A clause can be seen as a finite set of literals and a CNF formula as a finite set of clauses. A truth assignment for a CNF formula $F$ is a function $\tau$ that maps literals in $F$ to $\{0,1\}$. If $\tau(x)=v$, then $\tau(\bar{x})=1-v$. A clause $C$ is satisfied by $\tau$ if $\tau(l)=1$ for some literal $l \in C$. An assignment $\tau$ satisfies $F$ if it satisfies every clause in $F$.

Two formulas are logically equivalent if they are satisfied by exactly the same set of assignments. A clause is a tautology if it contains both $x$ and $\bar{x}$ for some variable $x$. The length of a clause is the number of literals in the clause. A clause of length one is a unit clause, and a clause of length two is a binary clause. For a CNF formula $F$, we denote the set of binary clauses in $F$ by $F_{2}$.

Binary Implication Graphs For any CNF formula $F$, we associate a unique directed binary implication graph $\mathrm{BIG}(F)$ with the edge relation $\left\{\left\langle\bar{l}, l^{\prime}\right\rangle,\left\langle\bar{l}^{\prime}, l\right\rangle \mid\left(l \vee l^{\prime}\right) \in F_{2}\right\}$. 
In other words, for each binary clause $\left(l \vee l^{\prime}\right)$ in $F$, the two implications $\bar{l} \rightarrow l^{\prime}$ and $\bar{l}^{\prime} \rightarrow l$, represented by the binary clause, occur as edges in $\operatorname{BIG}(F)$. A node in $\operatorname{BIG}(F)$ with no incoming arcs is a root of $\mathrm{BIG}(F)$ (or, simply, of $F_{2}$ ). In other words, literal $l$ is a root in $\operatorname{BIG}(F)$ if there is no clause of the form $\left(l \vee l^{\prime}\right)$ in $F_{2}$. The set of roots of $\operatorname{BIG}(F)$ is denoted by $\operatorname{RTS}(F)$.

\subsection{Known Simplification Techniques}

BCP and Failed Literal Elimination (FLE) For a CNF formula $F$, Boolean constraint propagation $(\mathrm{BCP})$ (or unit propagation) propagates all unit clauses, i.e. repeats the following until fixpoint: if there is a unit clause $(l) \in F$, remove from $F \backslash\{(l)\}$ all clauses that contain the literal $l$, and remove the literal $l$ from all clauses in $F$, resulting in the formula $\mathrm{BCP}(F)$. A literal $l$ is a failed literal if $\mathrm{BCP}(F \cup\{(l)\})$ contains the empty clause, implying that $F$ is logically equivalent to $\mathrm{BCP}(F \cup\{(\bar{l})\})$. FLE removes failed literals from a formula, or, equivalently, adds the complements of failed literals as unit clauses to the formula.

Equivalent Literal Substitution (ELS) The strongly connected components (SCCs) of $\mathrm{BIG}(F)$ describe equivalent classes of literals (or simply equivalent literals) in $F_{2}$. Equivalent literal substitution refers to substituting in $F$, for each SCC $G$ of $\operatorname{BIG}(F)$, all occurrences of the literals occurring in $G$ with the representative literal of $G$. ELS is confluent, i.e., has a unique fixpoint, modulo variable renaming.

Hidden Tautology Elimination (HTE) [6] For a given CNF formula $F$ and clause $C$, (hidden literal addition) $\operatorname{HLA}(F, C)$ is the unique clause resulting from repeating the following clause extension steps until fixpoint: if there is a literal $l_{0} \in C$ such that there is a clause $\left(l_{0} \vee l\right) \in F_{2} \backslash\{C\}$ for some literal $l$, let $C:=C \cup\{\bar{l}\}$. Note that $\operatorname{HLA}(F, C)=\operatorname{HLA}\left(F_{2}, C\right)$. Further, for any $l \in \operatorname{HLA}(F, C) \backslash C$, there is a path in $\operatorname{BIG}(F)$ from $l$ to some $l_{0} \in C$. For any CNF formula $F$ and clause $C \in F,(F \backslash$ $\{C\}) \cup\{\operatorname{HLA}(F, C)\}$ is logically equivalent to $F$ [6]. Intuitively, each extension step in computing HLA is an application of self-subsuming resolution [215]16] in reverse order. For a given CNF formula $F$, a clause $C \in F$ is a hidden tautology if and only if $\operatorname{HLA}(F, C)$ is a tautology. Hidden tautology elimination removes hidden tautologies from $\mathrm{CNF}$ formulas.

Note that distillation [4] is more generic than HTE [6] (and also more generic than HLE as defined in this paper). However, it is rather costly to apply, and is in practice restricted to irredundant/original clauses only.

Transitive reduction of the binary implication graph (TRD) A directed acyclic graph $G^{\prime}$ is a transitive reduction [11] of the directed graph $G$ provided that (i) $G^{\prime}$ has a directed path from node $u$ to node $v$ if and only if $G$ has a directed path from node $u$ to node $v$, and (ii) there is no graph with fewer edges than $G^{\prime}$ satisfying condition (i). It is interesting to notice that, by applying FLE restricted to the literals in $F_{2}$ before HTE, HTE achieves a transitive reduction of $\mathrm{BIG}(F)$ for any CNF formula $F$ purely on the clausal level [6]. 


\section{Hidden Literal Elimination}

In this section we present a novel redundancy elimination procedure exploiting the binary clause structure of a CNF formula. We call the technique hidden literal elimination.

For a given CNF formula $F$ and literal $l$, we denote by $\operatorname{HL}(F, l)$ the unique set of hidden literals of $l$ w.r.t $F$. $\operatorname{HL}(F, l)$ is defined as follows. First, let $L=\{l\}$. Then repeat the following steps until fixpoint: if there is a literal $l_{0} \in L$ such that there is a clause $\left(l_{0} \vee l^{\prime}\right) \in F_{2}$ for some literal $l^{\prime}$, let $L:=L \cup\left\{\bar{l}^{\prime}\right\}$. Now, let $\operatorname{HL}(F, l):=L \backslash\{l\}$. In other words, $\operatorname{HL}(F, l)$ contains the complements of all literals that are reachable from $\bar{l}$ in $\operatorname{BIG}(F)$, or, equivalently, all literals from which $l$ is reachable in $\operatorname{BIG}(F)$. Notice that $\operatorname{HL}(F, l)=\operatorname{HL}\left(F_{2}, l\right)$. Also, HL captures failed literals in $F_{2}$ in the sense that by definition, for any literal $l$ in $F_{2}$, there is a path from $l$ to $\bar{l}$ in $\operatorname{BIG}(F)$ if and only if $\bar{l} \in \operatorname{HL}(F, l)$.

Proposition 1. For any $C N F$ formula $F$, a literal $l$ in $F_{2}$ is failed iff $\bar{l} \in \operatorname{HL}(F, l)$.

For a given formula $F$, hidden literal elimination (HLE) repeats the following: if there is a clause $C \in F$ and a literal $l \in C$ such that $C \cap \operatorname{HL}(F, l) \neq \emptyset$, let $F:=$ $(F \backslash\{C\}) \cup\{C \backslash \operatorname{HL}(F, l)\}$. In fact, the literals in $\operatorname{HL}(F, l)$ can be removed from all clauses that contain $l$.

Proposition 2. For every CNF formula $F$, any result of applying HLE on $F$ is logically equivalent to $F$.

Proof. For any CNF formula $F$ and two literals $l$ and $l^{\prime}$, if $l^{\prime} \in \operatorname{HL}(F, l)$, then $F \cup\left\{\left(l^{\prime}\right)\right\}$ logically implies $l$ by the definition of HL. Hence, for any clause $C \in F$ with $l, l^{\prime} \in C$, for any satisfying assignment $\tau$ for $F$ with $\tau\left(l^{\prime}\right)=1$ we have $\tau(l)=1$, and hence $\tau$ satisfies $(F \backslash\{C\}) \cup\{C \backslash \mathrm{HL}(F, l)\}$.

A relevant question is how many literals HLE eliminates relative to other literal elimination techniques. One example is self-subsuming resolution (SSR) [2] that replaces clauses that have a resolvent that subsumes the clause itself with the resolvent (essentially eliminating from the clause the literal not in the resolvent).

Proposition 3. There are CNF formulas from which HLE can remove more literals from clauses than SSR.

Proof. Consider the formula $F=(a \vee b) \wedge(\bar{b} \vee c) \wedge(a \vee \bar{c} \vee d)$. Since $\operatorname{HL}(F, a)=\{\bar{b}, \bar{c}\}$, HLE can remove literal $\bar{c}$ from the last clause in contrast to SSR.

HLE can also strengthen formulas by increasing possibilities for unit propagation.

Proposition 4. Removal of hidden literals can increase BCP.

Proof. Consider the formula $F=(a \vee b) \wedge(\bar{b} \vee c) \wedge(a \vee \bar{c} \vee d)$. Since $\operatorname{HL}(F, a)=\{\bar{b}, \bar{c}\}$, HLE removes literal $\bar{c}$ from the last clause. When $d$ is assigned to 0 after eliminating literal $\bar{c}$, BCP will infer $a$.

In general, HLE does not have a unique fixpoint.

Proposition 5. Applying HLE until fixpoint is not confluent. 
Proof. Consider the formula $F=(a \vee b) \wedge(\bar{a} \vee \bar{b}) \wedge(a \vee \bar{b} \vee c)$. Since $\operatorname{HL}(F, a)=\{\bar{b}\}$ and $\operatorname{HL}(F, \bar{b})=\{a\}$, HLE can remove either $\bar{b}$ or $a$ from $(a \vee \bar{b} \vee c)$. A fixpoint is reached after removing one of these two literals.

In the example the non-confluence is due to $a$ and $\bar{b}$ being equivalent literals. In fact, assume that all clauses in $F_{2}$ are kept even in the case HLE turns a binary clause into a unit clause (i.e., in such cases HLE will introduce new unit clauses into $F$ ). Then HLE can be made confluent (modulo variable renaming) by substituting equivalent literals.

Theorem 1. For any $C N F$ formula $F$, assuming that all clauses in the original $F_{2}$ are kept, alternating ELS and HLE (until fixpoint) until fixpoint is confluent modulo variable renaming.

Proof sketch. ELS is confluent modulo variable renaming. Now consider HLE. Assume that we do not change $F_{2}$. Take any clause $C$ with $l, l^{\prime} \in C$ and $l^{\prime} \in \operatorname{HL}(F, l)$. The only possible source of non-confluence is that $l \in \operatorname{HL}\left(F, l^{\prime}\right)$. Then there is a cycle in $F_{2}$, and hence $l$ and $\bar{l}^{\prime}$ are equivalent literals. This is handled by ELS afterwards. Now assume a binary clause is added to $F_{2}$ by HLE shortening a clause of length $>2$. Newly produced cycles are handled by ELS afterwards.

\section{Unhiding Redundancies based on Time Stamping}

In this section we present an efficient algorithm for detecting several kinds of redundancies in CNF formulas, focusing on techniques which exploit binary clauses.

For a given CNF formula $F$, our algorithm, referred to as Unhiding (see Fig. 1 details explained in the following), consists in essence of two phases. First, a depth-first search (DFS) over the binary implication graph $\mathrm{BIG}(F)$ is performed. During the DFS, each literal in $\operatorname{BIG}(F)$ is assigned a time stamp; we call this process time stamping. In the second phase, these time stamps are used for discovering the various kinds of redundancies in $F$, which are then removed.

In the following, we will first describe a basic time stamping procedure (Sect.4.1). Then we will show how redundancies can be detected and eliminated based on the time stamps (Sect. 5). After these, in Sect. 6 we describe a more advanced time stamping procedure that embeds additional simplifications that are captured during the actual depth-first traversal of $\mathrm{BIG}(F)$.

\subsection{Basic Time Stamping}

The basic time stamping procedure implements a depth-first search on the binary implication graph $\mathrm{BIG}(F)$ of a given CNF formula $F$. The procedure associates a discoveredfinished interval (or a time stamp) with each literal in $\operatorname{BIG}(F)$ according to the depthfirst traversal order. For any depth-first traversal of a graph $G$, a node in $G$ is discovered (resp. finished) the first (resp. last) time it is encountered during search. For a given depth-first traversal, the discovery and finish times of a node $v$ in $G$, denoted by $\operatorname{dsc}(v)$ and $\operatorname{fin}(v)$, respectively, are defined as the number of steps taken at the time of discovering and finishing, respectively, the node $v$. The important observation here is that, according to the well-known "parenthesis theorem", for two nodes $u$ and $v$ with discovered-finished intervals $[\operatorname{dsc}(u), \operatorname{fin}(u)]$ and $[\operatorname{dsc}(v), \operatorname{fin}(v)]$, respectively, we 

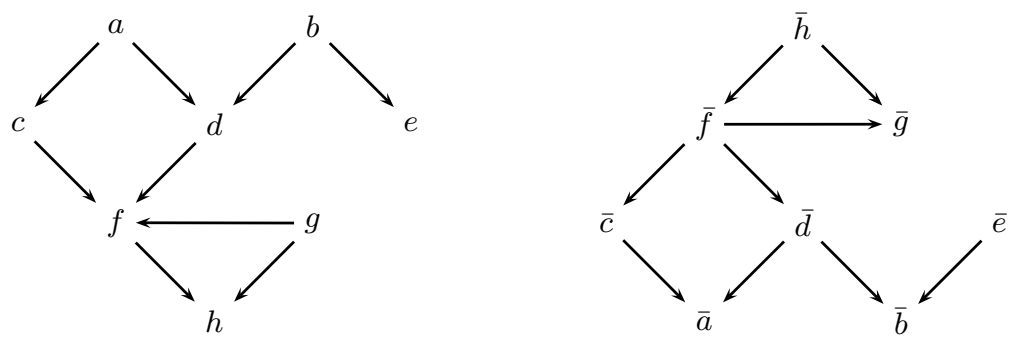

Fig. 2. $\operatorname{BIG}(E)$. The graph has five root nodes: $a, b, \bar{e}, g$, and $\bar{h}$.

The order in which the trees are traversed has a big impact on the quality, i.e. the fraction of implications that are represented by the time stamps. The example shows that randomized stamping may not represent all implications in BIG. Yet, for this formula, there is a DFS order that produces a stamping that represents all implications: start from the root $\bar{h}$ and stamp the tree starting with literal $\bar{f}$. Then, by selecting $a$ as the root of the second tree, regardless of the order of the other roots and literals, the time stamps produced by stamping will represent all implications.
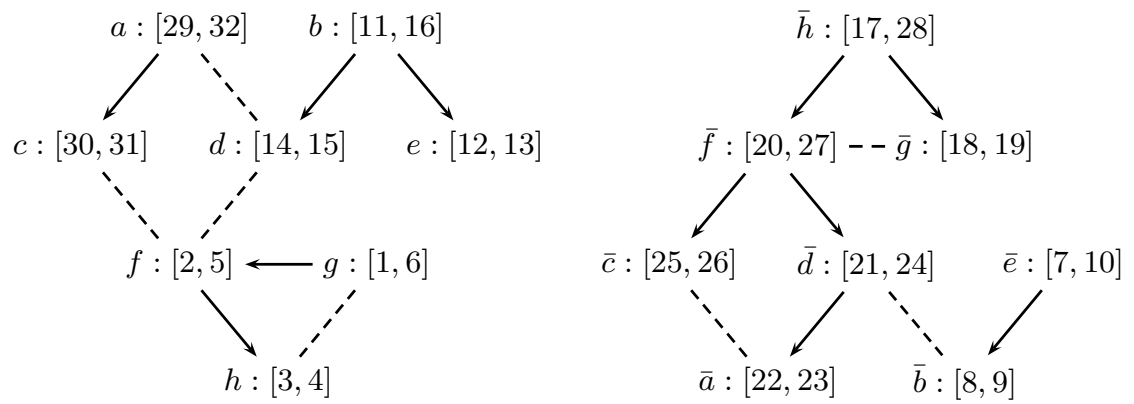

Fig. 3. A partition of $\operatorname{BIG}(E)$ into a forest with discovered-finished intervals $[\operatorname{dsc}(v), \operatorname{fin}(v)]$ assigned by the basic time stamping routine. Dashed lines represent implications in $\operatorname{BIG}(E)$ which are not used to set the time stamps.

\section{Capturing Various Simplifications}

We now explain how one can remove hidden literals and hidden tautologies, and furthermore perform hyper binary resolution steps based on a forest over the time stamped literal nodes produced by the main DFS procedure. The main procedure Simplify for this second phase, called by the main Unhiding procedure after time stamping, is shown in Fig. 4 For each clause $C$ in the input CNF formula $F$, Simplify removes $C$ from $F$. Then, it first checks whether the UHTE procedure detects that $C$ is a hidden tautology. If not, literals are (possibly) eliminated from $C$ by the $U H L E$ procedure (using hidden literal elimination). The resulting clause is added to $F$.

Notice that the simplification procedure visits each clause $C \in F$ only once. The invoked sub-procedures, UHTE and UHLE, exploit the time stamps, and use two sorted lists: (i) $S^{+}(C)$, list of the literals in $C$ sorted according to increasing discovery time, and (ii) $S^{-}(C)$, list of the complements of the literals in $C$, sorted according to increasing discovery time. We will now explain both of these sub-procedures in detail. 


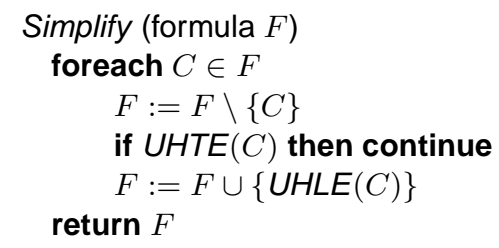

Fig. 4. Procedure for applying HTE and HLE based on time stamps.

\subsection{Hidden Literals}

Once literals are stamped using the unhiding algorithm, one can cheaply detect (possibly a subset of) hidden literals. In this context, literal $l \in C$ is hidden if there is (i) an implication $l \rightarrow l^{\prime}$ with $l^{\prime} \in C$ that is represented by the time stamping, or (ii) an implication $\bar{l}^{\prime} \rightarrow \bar{l}$ with $l^{\prime} \in C$ that is represented by the time stamping.

We check for such implications as follows using the UHLE procedure shown in Fig. 5 For each input clause $C$, the procedure returns a subset of $C$ with some hidden literals removed from $C$. For this procedure, we use $S^{+}(C)$ in reverse order, denoted by $S_{\text {rev }}^{+}(C)$. In essence, we go through the lists $S_{\text {rev }}^{+}(C)$ and $S^{-}(C)$, and compare the finish times of two successive elements in the lists. In case an implication is found, a hidden literal is detected and removed.

Lines 1-4 in Fig. 5 detect implications of the form $l \rightarrow l^{\prime}$ with $l, l^{\prime} \in C$ that are represented by the time stamping. Recall that in $S_{\mathrm{rev}}^{+}(C)$ literals are ordered with decreasing discovering time. Let $l^{\prime}$ be located before $l$ in $S_{\mathrm{rev}}^{+}(C)$. If $\operatorname{fin}(l)>\operatorname{fin}\left(l^{\prime}\right)$ we found the implication $l \rightarrow l^{\prime}$, and hence $l$ is a hidden literal (in the code finished $=$ fin $\left(l^{\prime}\right)$ ). Line 3 checks whether the next element in $S_{\text {rev }}^{+}(C)$ is a hidden literal, and if so, the literal is removed. Lines 5-8 detect implications $\bar{l}^{\prime} \rightarrow \bar{l}$ with $l, l^{\prime} \in C$. In $S^{-}(C)$ literals are ordered with increasing discovering time. Now, $\bar{l}^{\prime}$ be located before $\bar{l}$ in $S^{-}(C)$ and finished $=\operatorname{fin}\left(\bar{l}^{\prime}\right)$. On Line 7 we check that $\operatorname{fin}(\bar{l})<\operatorname{fin}\left(\bar{l}^{\prime}\right)$ or, equivalently, $\operatorname{fin}(\bar{l})<$ finished. In that case $l$ is a hidden literal and is hence removed.

Example 2. Recall the formula $E$ from Example 1 All literals except $e$ and $h$ in the clause $C=(a \vee b \vee c \vee d \vee e \vee f \vee g \vee h) \in E$ are hidden. In case the literals in $\operatorname{RTS}(E)$ are stamped with the time stamps shown in Figure 3, the UHLE procedure can detect them all. Consider first the sequence $S_{\mathrm{rev}}^{+}(C)=(c, a, d, e, b, h, f, g)$. Since $\operatorname{fin}(c)<\operatorname{fin}(a), a$ is removed from $C$. Similarly, fin $(e)<\operatorname{fin}(b)$ and $\operatorname{fin}(f)<\operatorname{fin}(g)$, and hence $b$ and $g$ are removed from $C$. Second, consider the complements of the literals in the reduced clause: $S^{-}(C)=(\bar{e}, \bar{h}, \bar{f}, \bar{d}, \bar{c})$. Now, fin $(\bar{h})>\operatorname{fin}(\bar{f}), \operatorname{fin}(\bar{d})$, fin $(\bar{c})$, and hence $f, d$, and $c$ are removed.

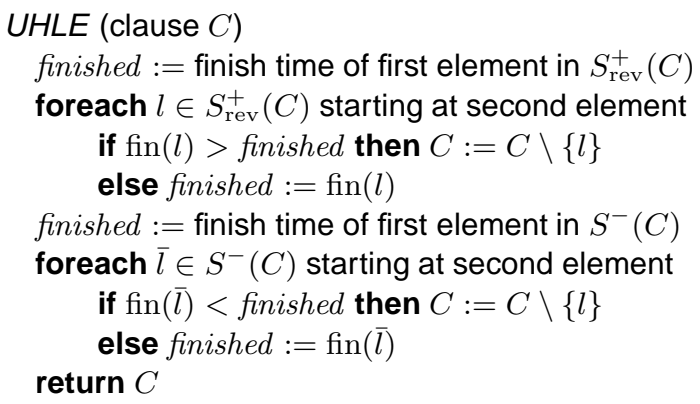

Fig. 5. Eliminating hidden literals using time stamps. 


\subsection{Hidden Tautologies}

Fig. 6] shows the pseudo-code for the UHTE procedure that detects hidden tautologies based on time stamps. Notice that if a time stamping represents an implication of the form $\bar{l} \rightarrow l^{\prime}$, where both $l$ and $l^{\prime}$ occur in a clause $C$, then the clause $C$ is a hidden tautology.

The UHTE procedure goes through the sorted lists $S^{+}(C)$ and $S^{-}(C)$ to find two literals $l_{\text {neg }} \in S^{-}(C)$ and $l_{\text {pos }} \in S^{+}(C)$ such that the time stamping represents the implication $l_{\text {neg }} \rightarrow l_{\text {pos }}$, i.e., it checks if $\operatorname{dsc}\left(l_{\text {neg }}\right)<\operatorname{dsc}\left(l_{\text {pos }}\right)$ and $\operatorname{fin}\left(l_{\text {neg }}\right)>\operatorname{fin}\left(l_{\text {pos }}\right)$. The procedure starts with the first literals $l_{\text {neg }} \in S^{-}(C)$ and $l_{\text {pos }} \in S^{+}(C)$, and loops through the literals in $l_{\text {pos }} \in S^{+}(C)$ until $\operatorname{dsc}\left(l_{\text {neg }}\right)<\operatorname{dsc}\left(l_{\text {pos }}\right)$ (Lines 4-6). Once such a $l_{\text {pos }}$ is found, if fin $\left(l_{\text {neg }}\right)>\operatorname{fin}\left(l_{\text {pos }}\right)$ (Line 7), we know that $C$ is a hidden tautology, and the procedure returns true (Line 10). Otherwise, we loop through $S^{-}(C)$ to select a new $l_{\text {neg }}$ for which the condition holds (Lines 7-9). Then (Lines 4-6), if $\operatorname{dsc}\left(l_{\text {neg }}\right)<\operatorname{dsc}\left(l_{\text {pos }}\right), C$ is a hidden tautology. Otherwise, we select a new $l_{\text {pos }}$. Unless a hidden tautology is detected, the procedure terminates once it has looped through all literals in either $S^{+}(C)$ or $S^{-}(C)$ (Lines 5 and 8).

One has to be careful while removing binary clauses based on time stamps. There are two exceptions in which time stamping represents an implication $l_{\text {neg }} \rightarrow l_{\text {pos }}$ with $l_{\text {neg }} \in S^{-}(C)$ and $l_{\text {pos }} \in S^{+}(C)$ for which $C$ is not a hidden tautology. First, if $l_{\text {pos }}=\bar{l}_{\text {neg }}$, then $l_{\text {neg }}$ is a failed literal. Second, if $\operatorname{prt}\left(l_{\text {pos }}\right)=l_{\text {neg }}$, then $C$ was used to set the time stamp of $l_{\text {pos }}$. Line 7 takes both of these cases into account.

Example 3. Recall again the formula $E$ from Example 1 contains three hidden tautologies: $(\bar{g} \vee h),(\bar{a} \vee \bar{e} \vee h)$, and $(\bar{b} \vee \bar{c} \vee h)$. In the time stamping in Fig. $3 \bar{h}:[17,28]$ contains $\bar{g}:[18,19]$. However, $\operatorname{prt}(\bar{g})=\bar{h}$, and hence $(\bar{g} \vee h)$ cannot be removed. On the other hand, $\bar{g}:[1,6]$ contains $\bar{h}:[3,4]$, and $\operatorname{prt}(h) \neq g$, and hence $(\bar{g} \vee h)$ is identified as a hidden tautology. We can also identify $(\bar{a} \vee \bar{e} \vee h)$ as a hidden tautology because $\bar{h}:[17,28]$ contains $\bar{a}:[22,23]$. This is not the case for $(\bar{b} \vee \bar{c} \vee h)$ because the implications $b \rightarrow h$ and $\bar{h} \rightarrow \bar{b}$ are not represented by the time stamping.

Proposition 6. For any Unhiding time stamping, UHTE detects all hidden tautologies that are represented by the time stamping.

Proof sketch. For every $l_{\text {neg }} \in S^{-}(C)$, UHTE checks if time stamping represents the implication $l_{\text {neg }} \rightarrow l_{\text {pos }}$ for the first literal in $l_{\text {pos }} \in S^{+}(C)$ for which $\operatorname{dsc}\left(l_{\text {neg }}\right)<$

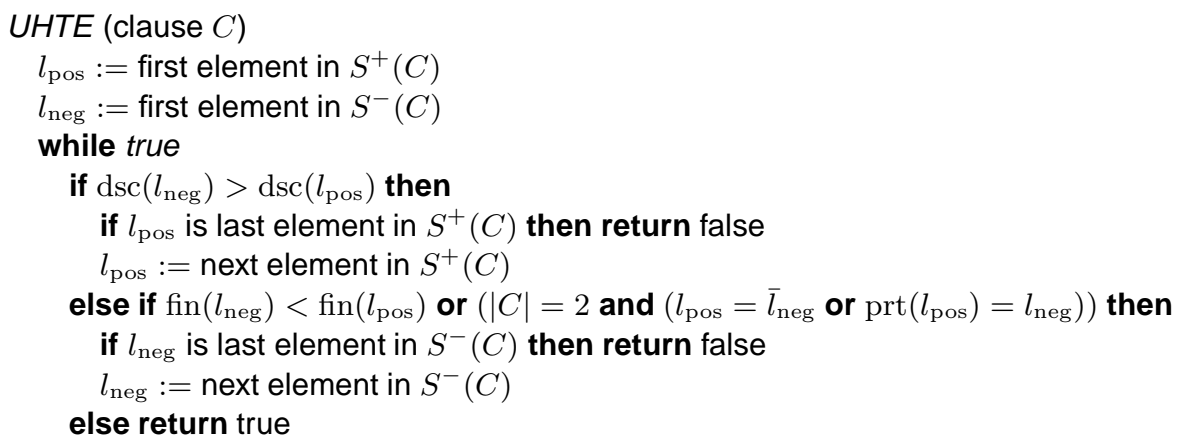

Fig. 6. Detecting hidden tautologies using time stamps. 
$\operatorname{dsc}\left(l_{\text {pos }}\right)$ holds. The key observation is that if there is a $l_{\text {neg }} \in S^{-}(C)$ and a $l_{\text {pos }} \in$ $S^{+}(C)$ such that time stamping represents the implication $l_{\text {neg }} \rightarrow l_{\text {pos }}$, then the stamps also represent $l_{\text {neg }} \rightarrow l_{\text {pos }}^{\prime}$ with $l_{\text {pos }}^{\prime}$ being the first literal in $S^{+}(C)$ for which $\operatorname{dsc}\left(l_{\text {neg }}\right)<$ $\operatorname{dsc}\left(l_{\text {pos }}\right)$ holds.

If a clause $C$ is a hidden tautology, then $\operatorname{HLA}(F, C)$ is a hidden tautology due to $\operatorname{HLA}(F, C) \supseteq C$. However, it is possible that, for a given clause $C, \operatorname{UHTE}(C)$ returns true, while $U H T E(U H L E(C))$ returns false. In other words, $U H L E$ could in some cases disrupt UHTE. For instance, consider the clause $(a \vee b \vee c)$ and the following time stamps: $a:[2,3], \bar{a}:[9,10], b:[1,4], \bar{b}:[5,8], c:[6,7], \bar{c}:[11,12]$. Now UHLE removes literal $b$ which is required for UHTE to return true. Therefore UHTE should be called before UHLE, as is done in our Simplify procedure (recall Fig. 4).

\subsection{Adding Hyper Binary Resolution}

An additional binary clause based simplification technique that can be integrated into the unhiding procedure is hyper binary resolution [1] (HBR). Given a clause of the form $\left(l_{1} \vee \cdots \vee l_{k}\right)$ and $k-1$ binary clauses of the form $\left(l^{\prime} \vee \bar{l}_{i}\right)$, where $2 \leq i \leq k$, the hyper binary resolution rule allows to infer the clause $\left(l_{1} \vee l^{\prime}\right)$ in one step.

For HBR in the unhiding algorithm we only need the list $S^{-}(C)$. Let $C$ be a clause with $k$ literals. We find a hyper binary resolvent if (i) all literals in $S^{-}(C)$, except the first one $l_{1}$, have a common ancestor $l^{\prime}$, or (ii) all literals in $S^{-}(C)$, except the last one $l_{k}$, have a common ancestor $l^{\prime \prime}$. In case (i) we find $\left(l_{1} \vee \bar{l}^{\prime}\right)$, and in case (ii) we find $\left(l_{k} \vee \bar{l}^{\prime \prime}\right)$. It is even possible that all literals in $S^{-}(C)$ have a common ancestor $l^{\prime \prime \prime}$ which shows that $l^{\prime \prime \prime}$ is a failed literal, in which case we can learn the unit clause $\left(\bar{l}^{\prime \prime \prime}\right)$.

While $\operatorname{UHBR}(C)$ could be called in Simplify after Line 4, our experiments show that applying $\operatorname{UHBR}(C)$ does not give further gains w.r.t. running times, and can in cases degrade performance. We suspect that this is because $\operatorname{UHBR}(C)$ may add transitive edges to $\operatorname{BIG}(F)$. Consider the formula $F=(a \vee b \vee c) \wedge(\bar{a} \vee d) \wedge(\bar{b} \vee d) \wedge(c \vee e) \wedge$ $(c \vee f) \wedge(d \vee \bar{e})$. Assume that the time stamping DFS visits the literals in the order $\bar{f}$, $c, a, d, \bar{d}, \bar{e}, \bar{a}, \bar{b}, \bar{c}, f, e, b . U H B R((a \vee b \vee c))$ can learn $(c \vee d)$, but it cannot check that this binary clause adds a transitive edge to $\operatorname{BIG}(F)$.

\subsection{Some Limitations of Basic Stamping}

As already pointed out, time stamps produced by randomized DFS may not represent all implications of $F_{2}$. In fact, the fraction of implications represented can be very small in the worst case. Especially, consider the formula $F=(a \vee b \vee c \vee d) \wedge(\bar{a} \vee \bar{b}) \wedge(\bar{a} \vee$ $\bar{c}) \wedge(\bar{a} \vee \bar{d}) \wedge(\bar{b} \vee \bar{c}) \wedge(\bar{b} \vee \bar{d}) \wedge(\bar{c} \vee \bar{d})$ that encodes that exactly one of $a, b, c, d$ must be true. Due to symmetry, there is only one possible DFS traversal order, and it produces the time stamps $a:[1,8], \bar{b}:[2,3], \bar{c}:[4,5], \bar{d}:[6,7], b:[9,12], \bar{a}:[10,11], c:$ $[13,14], d:[15,16]$. Only three of the six binary clauses are represented by the time stamps. This example can be extended to $n$ variables, in which case only $n-1$ of the $n(n-1) / 2$ binary clauses are represented. In order to capture as many implications (and thus simplification opportunities) as possible, in practice we apply multiple repetitions of Unhiding using randomized DFS (as detailed in Sect. (7). 


\section{Advanced Stamping for Capturing Additional Simplifications}

In this section we develop an advanced version of the DFS time stamping procedure. Our algorithm can be seen as an extension of the BinSATSCC-1 algorithm in [13]. The advanced procedure, presented in Fig. 7 enables performing additional simplifications on-the-fly during the actual time stamping phase: the on-the-fly techniques can perform some simplifications that cannot be done with $\operatorname{Simplify}(F)$, and, on the other hand, enlarging the time stamps of literals may allow further simplifications in Simplify $(F)$. Although not discussed further in this paper due to the page limit, we note that, additionally, all simplifications by UHTE, UHLE, and UHBR which only use binary clauses could be performed on-the-fly within the advanced stamping procedure.

Here we introduce the attribute obs $(l)$ that denotes the latest time point of observing $l$. The value of obs $(l)$ can change frequently during Unhiding. Each line of the advanced stamping procedure (Fig. 7 is labeled. The line labeled with OBS assigns obs $(l)$ for literal $l$. The label BSC denotes that the line originates from the basic stamping procedure (Fig. 1). Lines with the other labels are techniques that can be performed on-thefly: transitive reduction (TRD / Sect. 6.1), failed literal elimination (FLE / Sect. 6.2, and equivalent literal substitution (ELS / Sect. 6.3). The technique TRD depends on FLE and both techniques use the obs() attribute while ELS is independent of obs().

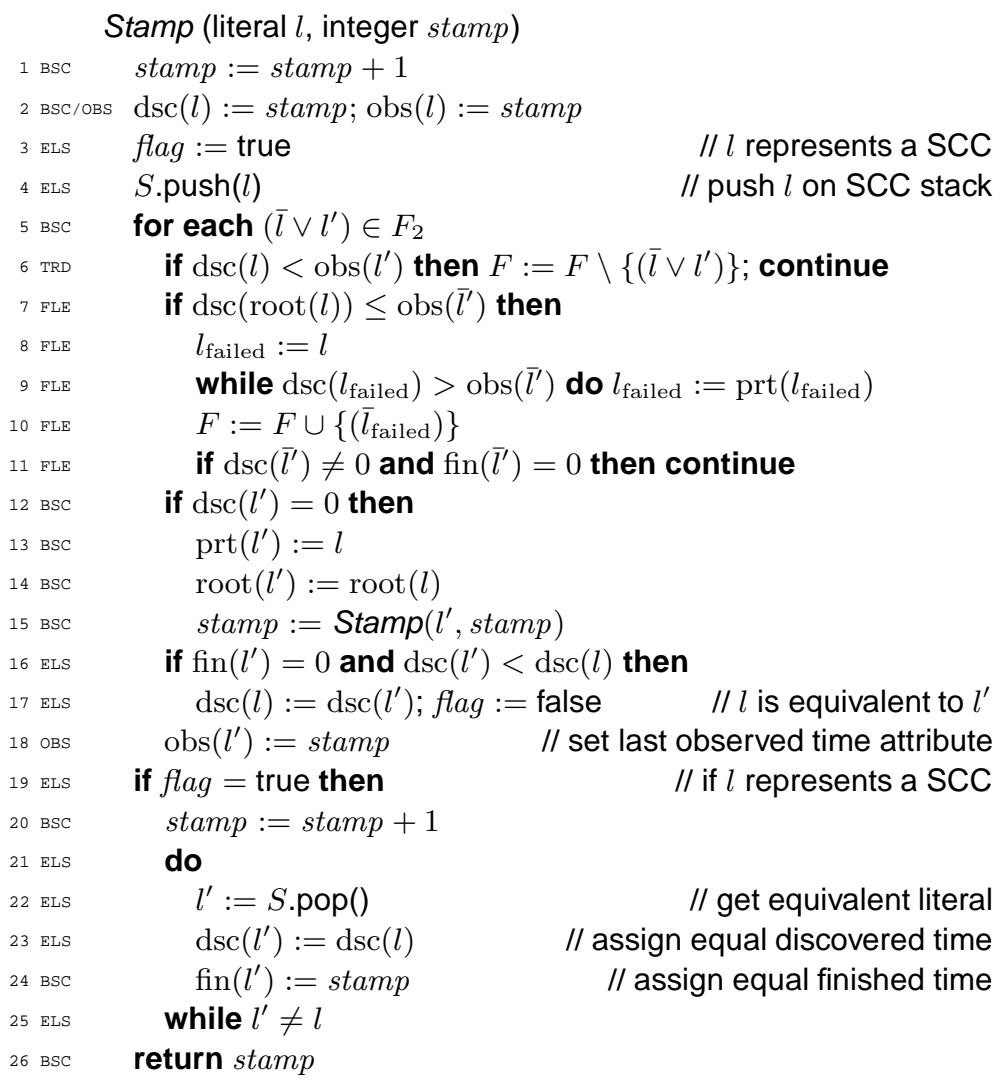

Fig. 7. Advanced literal time stamping capturing failed and equivalent literals 


\subsection{Transitive Reduction}

Binary clauses that represent transitive edges in BIG are in fact hidden tautologies [6]. Such clauses can already be detected in the stamping phase (i.e., before UHTE), as shown in the advanced stamping procedure on Line 6 with label TRD.

A binary clause $\left(\bar{l} \vee l^{\prime}\right)$ can only be observed as a hidden tautology if $\operatorname{dsc}\left(l^{\prime}\right)>0$ during $\operatorname{Stamp}(l, s t a m p)$. Otherwise, $\operatorname{prt}\left(l^{\prime}\right):=l$, which satisfies the last condition on Line 7 of $U H T E$. If $\operatorname{dsc}\left(l^{\prime}\right)>\operatorname{dsc}(l)$ just before calling $\operatorname{Stamp}\left(l^{\prime}, \operatorname{stamp}\right)$, then $\left(\bar{l} \vee l^{\prime}\right)$ is a hidden tautology. When transitive edges are removed on-the-fly, UHTE can focus on clauses of size $\geq 3$, making the last check on Line 7 of UHTE redundant.

Transitive edges in $\mathrm{BIG}(F)$ can hinder the unhiding algorithm by reducing the time stamp intervals. Hence as many transitive edges as possible should be removed. Notice that in case $0<\operatorname{dsc}\left(l^{\prime}\right)<\operatorname{dsc}(l), \operatorname{Stamp}(l, \operatorname{stamp})$ cannot detect that $\left(\bar{l} \vee l^{\prime}\right)$ is a hidden tautology. Yet by using obs $\left(l^{\prime}\right)$ instead of $\operatorname{dsc}\left(l^{\prime}\right)$ in the check (Line 14 of Fig. 7), we can detect additional transitive edges. For instance, consider the formula $F=(\bar{a} \vee b) \wedge$ $(b \vee \bar{c}) \wedge(b \vee \bar{d}) \wedge(\bar{c} \vee d)$ where $(b \vee \bar{c})$ is a hidden tautology. If Unhiding visits the literals in the order $a, b, c, d, \bar{b}, \bar{a}, \bar{c}, \bar{d}$, then this hidden tautology is not detected using $\operatorname{dsc}\left(l^{\prime}\right)$. However, while visiting $d$ in advanced stamping, we assign obs $(b):=\operatorname{dsc}(d)$. Now, using obs $\left(l^{\prime}\right), \operatorname{Stamp}(c$, stamp $)$ can detect that $(b \vee \bar{c})$ is a hidden tautology.

\subsection{Failed Literal Elimination over $\boldsymbol{F}_{\mathbf{2}}$}

Detection of failed literals in $F_{2}$ can be performed on-the-fly during stamping. If a literal $l$ in $F_{2}$ is failed, then all ancestors of $l$ in $\operatorname{BIG}(F)$ are also failed. Recall that there is a strong relation between HLE restricted to $F_{2}$ and failed literals in $F_{2}$ (Prop.11).

To detect a failed literal, we check for each observed literal $l^{\prime}$ whether $\bar{l}^{\prime}$ was also observed in the current tree, or $\operatorname{dsc}(\operatorname{root}(l)) \leq \operatorname{dsc}\left(\bar{l}^{\prime}\right)$. In that case the lowest common ancestor in the current tree is a failed literal. Similar to transitive reduction, the number of detected failed literals can be increased by using the obs $\left(\bar{l}^{\prime}\right)$ attribute instead of $\operatorname{dsc}\left(\bar{l}^{\prime}\right)$. We compute the lowest common ancestor $l_{\text {failed }}$ of $l^{\prime}$ and $\bar{l}^{\prime}$ (Lines 8-9 in Fig. (7). Afterwards the unit clause $\left(\bar{l}_{\text {failed }}\right)$ is added to the formula (Line 10).

At the end of on-the-fly FLE (Line 11), the advanced stamping procedure checks whether to stamp $l^{\prime}$ after finding a failed literal. In case we learned that $\bar{l}^{\prime}$ is a failed literal, then we have the unit clause $\left(l^{\prime}\right)$. Then it does not make sense to stamp $l^{\prime}$, as all implications of $l^{\prime}$ can be assigned to true by BCP. This check also ensures that binary clauses currently used in the recursion are not removed by transitive reduction.

\subsection{Equivalent Literal Substitution}

In case $\mathrm{BIG}(F)$ contains a cycle, then all literals in that cycle are equivalent. In the basic stamping procedure all these literals will be assigned a different time stamp. Therefore, many implications of $F_{2}$ will not be represented by any of the resulting time stampings. To fix this problem, equivalent literals should be assigned the same time stamps.

A cycle in $\operatorname{BIG}(F)$ can be detected after calling $\operatorname{Stamp}\left(l^{\prime}, \operatorname{stamp}\right)$, by checking whether fin $\left(l^{\prime}\right)$ still has the initial value 0 . This check can only return true if $l^{\prime}$ is an ancestor of $l$. We implemented ELS on-the-fly using a variant of Tarjan's SCC decomposition algorithm [17] which detects all cycles in $\mathrm{BIG}(F)$ using any depth-first traversal order. We use a local boolean flag that is initialized to true (Line 3). If true, 
flag denotes that $l$ represents a SCC. In case it detects a cycle, flag is set to false (Lines 16-17). Additionally, a global stack $S$ of literals is used, and is initially empty. At each call of $\operatorname{Stamp}(l, s t a m p), l$ is pushed on the stack (Line 4). At the end of the procedure, if $l$ is still the representative of a SCC, all literals in $S$ being equivalent to $l$, all literals in $S$ are assigned the same time stamp (Lines 19-25).

\section{Experiments}

We have implemented Unhiding in our state-of-the-art SAT solver Lingeling [12] (version 517, source code and experimental data at http://fmv.jku.at/unhiding) as an additional preprocessing or, more precisely, inprocessing technique applied during search. Batches of randomized unhiding rounds are interleaved with search and other already included inprocessing techniques. The number of unhiding rounds per unhiding phase and the overall work spent in unhiding is limited in a similar way as is already done in Lingeling for the other inprocessing. The cost of Unhiding is measured in the number of recursive calls to the stamping procedure and the number of clauses traversed. Sorting clauses (in UHTE and UHLE) incurs an additional penalty. In the experiments Unhiding takes on average roughly $7 \%$ of the total running time (including search), which is more than twice as much as standard failed literal probing $(2 \%)$ and around half of the time spent on SatElite-style variable elimination $(16 \%)$.

The cluster machines used for the experiments, with Intel Core 2 Duo Quad Q9550 2.8-GHz processors, 8-GB main memory, running Ubuntu Linux version 9.04, are around twice as fast as the ones used in the first phase of the 2009 SAT competition. For the experiments we used a $900 \mathrm{~s}$ timeout and a memory limit of 7 GB. Using the set of all 292 application instances from SAT Competition 2009 (http://satcompetition.org/2009/), a comparison of the number of solved instances for different configurations of Unhiding and the baseline (up-to-date version of Lingeling without Unhiding) is presented in Table 1 Note that we obtained similar results also for the SAT Race 2010 instances, and also improved performance on the crafted instances of SAT Competition 2009.

Table 1. Comparison of different configurations of Unhiding and the baseline solver Lingeling. The $2^{\text {nd }}$ to $4^{\text {th }}$ columns show the number of solved instances (sol), resp. solved satisfiable (sat) and unsatisfiable (uns) instances. The next three columns contain the average percentage of total time spent in unhiding (unhd), all simplifications through inprocessing (simp), and variable elimination (elim). Here we also take unsolved instances into account. The rest of the table lists the number of hidden tautologies (hte) in millions, the number of hidden literal eliminations (hle), also in millions, and finally the number of unhidden units (unts) in thousands which includes the number of unhidden failed literals. We also include the average percentage (stp) of hidden tautologies resp. derived units during stamping, and the average percentage (red) of redundant/learned hidden tautologies resp. removed literals in redundant/learned clauses. A more detailed analysis shows that for many instances, the percentage of redundant clauses is very high, actually close to $100 \%$, both for HTE and HLE. Note that "unts" is not precise as the same failed literal might be found several times during stamping since we propagate units lazily after unhiding.

\begin{tabular}{|c|c|c|c|c|c|c|c|c|c|c|c|c|c|}
\hline configuration & sol & sat| & uns & |lunhd & $\operatorname{simp}$ & elim & hte & stp & red & hle & red & unts & stp \\
\hline adv.stamp (no uhbr) & 188 & 78 & 110 & $7.1 \%$ & $33.0 \%$ & $16.1 \%$ & 22 & $64 \%$ & $59 \%$ & 291 & $77.6 \%$ & 935 & $57 \%$ \\
\hline adv.stamp (w/uhbr) & 184 & 75 & 109 & $7.6 \%$ & $32.8 \%$ & $15.8 \%$ & 26 & $67 \%$ & $70 \%$ & 278 & $77.9 \%$ & 941 & $58 \%$ \\
\hline basic stamp (no uhbr) & 183 & 73 & 110 & $6.8 \%$ & $32.3 \%$ & $15.8 \%$ & 6 & $0 \%$ & $52 \%$ & 296 & $78.0 \%$ & 273 & $0 \%$ \\
\hline basic stamp (w/uhbr) & 183 & 73 & 110 & $7.4 \%$ & $32.8 \%$ & $15.8 \%$ & 7 & $0 \%$ & $66 \%$ & 288 & $76.7 \%$ & 308 & $0 \%$ \\
\hline no unhiding & 180 & 74 & 106 & $0.0 \%$ & $28.6 \%$ & $17.6 \%$ & 0 & $0 \%$ & $0 \%$ & 00 & $0.0 \%$ & 0 & $0 \%$ \\
\hline
\end{tabular}


The three main observations are: (i) Unhiding increases the number of solved satisfiable instances already when using the basic stamping procedure; (ii) using the advanced stamping scheme, the number of solved instances increases notably for both satisfiable and unsatisfiable instances; and (iii) the $U H B R$ procedure actually degrades the performance (in-line with the discussion in Sect. 5.3. Hence the main advantages of Unhiding are due to the combination of the advanced stamping procedure, UHTE, and UHLE.

\section{Conclusions}

The Unhiding algorithm efficiently (close to linear time) approximates a combination of binary clause based simplifications that is conjectured to be at least quadratic in the worst case. In addition to applying known simplification techniques, including the recent hidden tautology elimination, we introduced the novel technique of hidden literal elimination, and implemented it within Unhiding. We showed that Unhiding improves the performance of a state-of-the-art CDCL SAT solver when integrated into the search procedure for inprocessing formulas (including learnt clauses) during search.

\section{References}

1. Bacchus, F.: Enhancing Davis Putnam with extended binary clause reasoning. In: Proc. AAAI, AAAI Press (2002) 613-619

2. Eén, N., Biere, A.: Effective preprocessing in SAT through variable and clause elimination. In: Proc. SAT. Volume 3569 of LNCS., Springer (2005) 61-75

3. Gershman, R., Strichman, O.: Cost-effective hyper-resolution for preprocessing CNF formulas. In: Proc. SAT. Volume 3569 of LNCS., Springer (2005) 423-429

4. Han, H., Somenzi, F.: Alembic: An efficient algorithm for CNF preprocessing. In: Proc. DAC, IEEE (2007) 582-587

5. Järvisalo, M., Biere, A., M. Heule, M.J.H.: Blocked clause elimination. In: Proc. TACAS. Volume 6015 of LNCS., Springer (2010) 129-144

6. Heule, M.J.H., Järvisalo, M., Biere, A.: Clause elimination procedures for CNF formulas. In: Proc. LPAR-17. Volume 6397 of LNCS., Springer (2010) 357-371

7. Marques Silva, J.P.: Algebraic simplification techniques for propositional satisfiability. In: Proc. CP. Volume 1894 of LNCS., Springer (2000) 537-542

8. Van Gelder, A.: Toward leaner binary-clause reasoning in a satisfiability solver. Annals of Mathematics and Artificial Intelligence 43(1) (2005) 239-253

9. Li, C.M.: Integrating equivalency reasoning into Davis-Putnam procedure. In: Proc. AAAI. (2000) 291-296

10. Brafman, R.: A simplifier for propositional formulas with many binary clauses. IEEE Transactions on Systems, Man, and Cybernetics, Part B 34(1) (2004) 52-59

11. Aho, A., Garey, M., Ullman, J.: The transitive reduction of a directed graph. SIAM Journal on Computing 1(2) (1972) 131-137

12. Biere, A.: Lingeling, Plingeling, PicoSAT and PrecoSAT at SAT Race 2010. FMV Report Series Technical Report 10/1, Johannes Kepler University, Linz, Austria (2010)

13. del Val, A.: Simplifying binary propositional theories into connected components twice as fast. In: Proc. LPAR. Volume 2250 of LNCS., Springer (2001) 392-406

14. Soos, M.: Cryptominisat 2.5.0, sat race 2010 solver description (2010)

15. Korovin, K.: iProver - an instantiation-based theorem prover for first-order logic. In: Proc. IJCAR. Volume 5195 of LNCS., Springer (2008) 292-298

16. Groote, J.F., Warners, J.P.: The propositional formula checker HeerHugo. J. Autom. Reasoning 24(1/2) (2000) 101-125

17. Tarjan, R.: Depth-first search and linear graph algorithms. SIAM J. Computing 1(2) (1972) 